\title{
A Closed-Loop Optimized System with CFD Data for Liquid Maldistribution Model
}

\author{
Wei Zhang *, Liyi Li, Baoping Zhang, Xin Xu, Jian Zhai and Junwen Wang
}

Department of Chemical Engineering, Taiyuan University of Technology, Taiyuan 030024, China; liliyi0448@link.tyut.edu.cn (L.L.); zhangbaoping0451@link.tyut.edu.cn (B.Z.); xuxin0457@link.tyut.edu.cn (X.X.); zhaijian0453@link.tyut.edu.cn (J.Z.); wangjunwen@tyut.edu.cn (J.W.)

* Correspondence: zhangwei01@tyut.edu.cn

Received: 24 September 2020; Accepted: 20 October 2020; Published: 22 October 2020

\begin{abstract}
For the simulation of a trickle-bed reactor (TBR) in coal and oil refining, modeling the liquid maldistribution of the gas-liquid distributor incurs enormous pre-processing work and bears a huge computational cost. A closed-loop optimized system with computational fluid dynamic (CFD) data is therefore proposed for the first time in this paper. A fast prediction model based on support vector regression (SVR) is developed to simplify the modeling of the liquid flow rate in TBRs. The model uses CFD simulation results to determine an optimized set of structural parameters for the gas-liquid distributor in TBRs. In order to obtain an accurate SVR model quickly, the particle swarm optimization (PSO) algorithm is employed to optimize the SVR parameters. Then, the structural parameters corresponding to the minimum liquid maldistribution factor are calculated using the response surface methodology (RSM) based on the hybrid PSO-SVR model. The CFD validation results show a good agreement with the values predicted by RSM, with liquid maldistribution factors of 0.159 and 0.162 , respectively.
\end{abstract}

Keywords: support vector regression (SVR); particle swarm optimization (PSO); computational fluid dynamic (CFD) simulation; liquid maldistribution model; gas-liquid distributor; response surface method (RSM)

\section{Introduction}

Trickle-bed reactors (TBRs) are widely used in the chemical and oil industries. TBR efficiency relies on the good distribution of liquid feed over the catalyst bed cross section, so predicting liquid maldistribution is one of the critical issues in the efficient use of TBRs [1].

Several approaches have been developed to estimate liquid maldistribution. Cold model experiments are easy to implement and relatively simple to perform in laboratory-scale devices using a collector at the catalyst bed outlet. However, there is a possibility of flow redistribution at the exit of the bed [2-4]. To overcome this disadvantage, several groups have focuses on the use of tomographic measurements using photon attenuation (x-ray and $\gamma$-ray tomography), magnetic resonance imagining, and electric tomographic techniques, which provide more quantitative flow distribution information [5-9]. With the drawback of a high cost and unsafety, the performance of these techniques depends on the complexity of the reconstruction algorithms. Compared to these approaches, computational fluid dynamics (CFD) modeling has a relatively low cost and can simulate both realistic and ideal conditions [10-14]. However, a CFD simulation of the full geometry of a gas-liquid distributor may be time-consuming, depending on the domain size and the mesh number. Hence, a time-saving and reliable surrogate model is needed for CFD simulation.

The support vector regression (SVR) method is such a tool generally preferred for solving problems requiring high computational resources [15-18]. Recently, the applicability of SVR-based models in the 
field of chemical engineering has been well demonstrated. SVR models have already been applied in predicting the behavior of the gas-liquid interface area $(a), k_{L a}$, and $k_{G a}$ in TBRs [19] by Bansal et al. Jalalifar et al. proposed an SVR-particle swarm optimization (PSO) model and combined it with a CFD dataset to predict the best operating parameters of a pyrolysis reactor [20]. Zaidi used an SVR-based modeling technique to predict the cycle rates in a thermosiphon reboiler for different pure components with large variations in thermophysical properties and operating parameters [21].

In this paper, we propose a methodology for obtaining an optimized solution of the gas-liquid distributor in TBRs on the basis of limited data generated using CFD simulation in order to reduce the simulation time significantly. The flow chart for obtaining an optimized solution of the gas-liquid distributor in TBRs is shown in Figure 1. In step 1, CFD data is used as the input to develop an SVR model, and then PSO is used to accelerate the training speed to find the optimum parameter set for the SVR model. In step 2, the response surface method (RSM) is used to optimize the structure of the gas-liquid distributor according to the PSO-SVR model, and then the optimum structure is validated through CFD simulation.

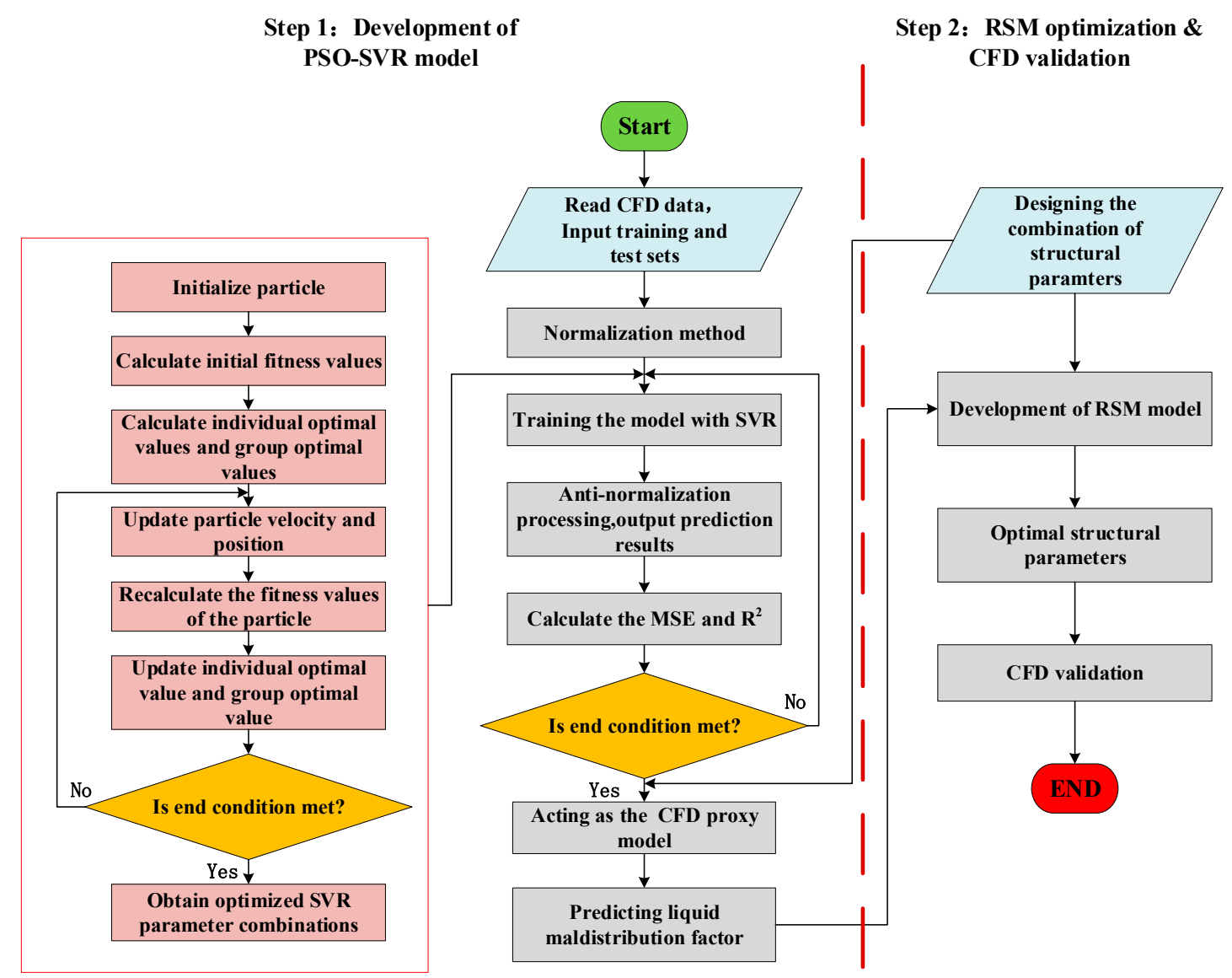

Figure 1. The flow chart for obtaining an optimized solution of the gas-liquid distributor in trickle-bed reactors (TBRs).

The remainder of this paper is organized as follows. In Section 2, a hybrid modeling method of PSO-SVR based on CFD simulation data is proposed. In Section 3, the optimal structure of the gas-liquid distributor is obtained via RSM on the basis of the data from the PSO-SVR model. In Section 4, a comparison is carried out to evaluate the universality, accuracy, and rapidity of PSO-SVR model, and the optimization result is verified by CFD simulation. Finally, we draw conclusions and describe the future research directions in Section 5. 


\section{SVR Modeling Hybrid PSO Based on CFD Simulation}

The present study aims to predict the liquid flow rate by training an SVR model of the TBR gas-liquid distributor with data obtained from CFD simulations. In order to accelerate the training speed of the SVR, PSO then is employed to optimize the parameters of the SVR, due to its efficiency in solving multidimensional problems.

\subsection{Data from CFD Simulations}

114 CFD simulation datasets extracted from previously published articles [22] were utilized in this study. The structure of the Venturi suction-type gas-liquid distributor [22] is shown in Figure 2, with a throat diameter $\mathrm{D}$, a throat height $\mathrm{H}$, and a throat length $\mathrm{L}$. To accurately show the uniformity of liquid distribution quantitatively, the liquid maldistribution factor $\sigma$ is defined according to the following Equation [2]:

$$
\sigma=\sqrt{\frac{1}{N(N+1)} \sum_{i=1}^{N}\left(\frac{u_{L, i}-\bar{u}_{L}}{\bar{u}_{L}}\right)^{2}}
$$

where $u_{L, i}$ is the liquid flow rate in zone $i, N$ is the number of zones (11 in this case), and $\bar{u}_{L}$ is the mean flow rate of all zones. As defined, the liquid maldistribution factor varies from 0 (representing the ideal distribution) to 1 (where all liquid flows into a certain region). Thus, a small maldistribution factor value qualifies a better distribution. The liquid flow rate is associated with the sampling plane position and the radial position of the trickle bed. In this paper, we assume that the catalyst bed is located $300 \mathrm{~mm}$ below the gas-liquid distributor plane (i.e., the sampling plane). Velocity sampling is performed at the center of the sampling plane, located at $0 \mathrm{~mm}$, and 5 radius positions $(30 \mathrm{~mm}, 50 \mathrm{~mm}$, $70 \mathrm{~mm}, 90 \mathrm{~mm}$, and $110 \mathrm{~mm}$ ), for a total of 6 positions. Hence, the radial radius is also used as an input variable of SVR.

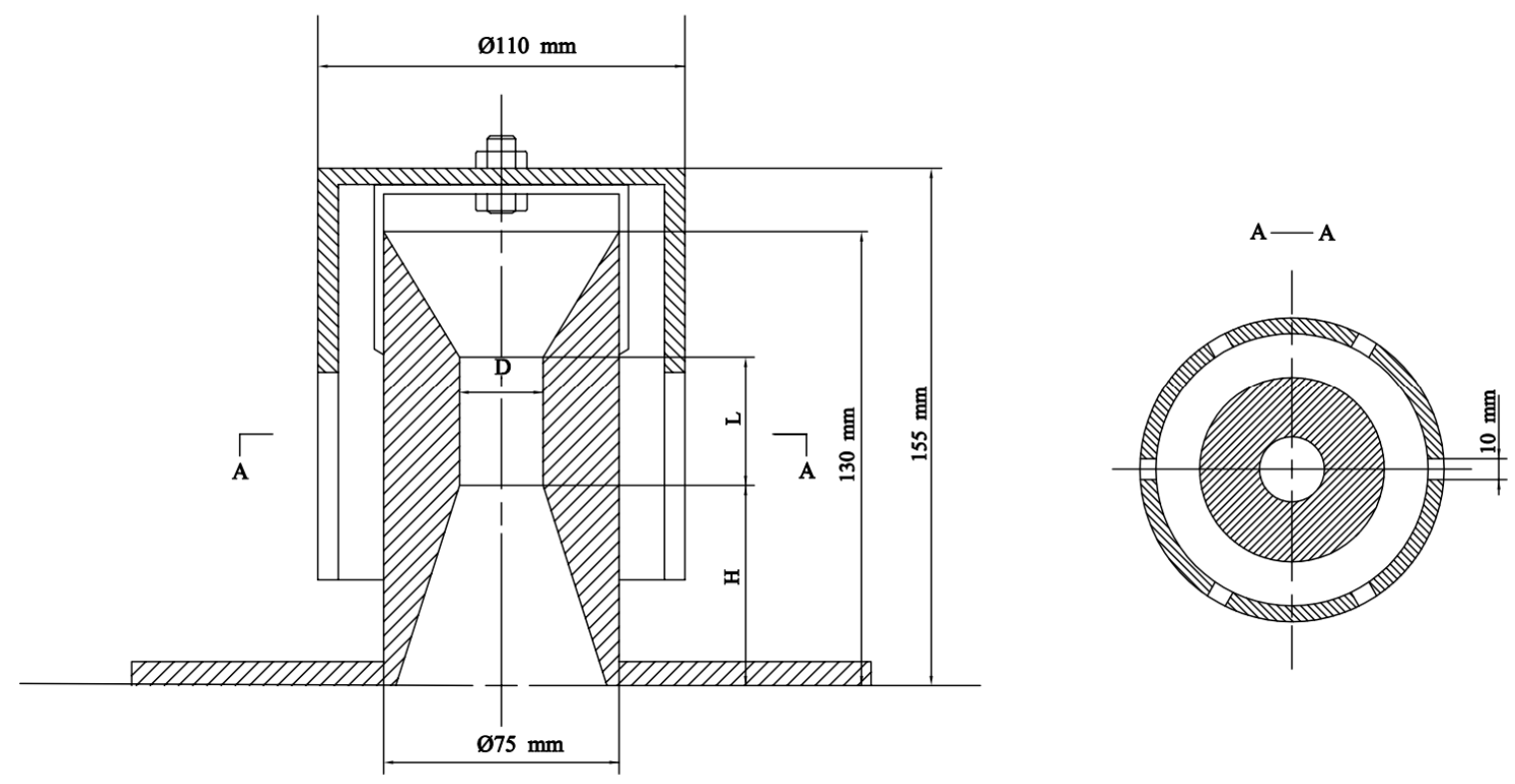

Figure 2. Structure of Venturi suction-type gas-liquid distributor.

In summary, there are four input variables: the throat diameter $(\mathrm{D})$, the throat height $(\mathrm{H})$, the throat length $(\mathrm{L})$, and radial radius $(\mathrm{R})$. The liquid flow rate through zone $i\left(u_{L, i}\right)$ is used as the output variable, and the maldistribution factor $\sigma$ as the indirect output variable. The input and output variables of the SVR model and part of the corresponding simulation data from the CFD are summarized in Table 1. 
Table 1. Input and output variables of the support vector regression (SVR) model.

\begin{tabular}{cccccc}
\hline & \multicolumn{2}{c}{ Input Variables } & \multicolumn{3}{c}{ Output Variables } \\
\hline $\boldsymbol{x}_{\mathbf{1}}$ & $\boldsymbol{x}_{\mathbf{2}}$ & $\boldsymbol{x}_{\mathbf{3}}$ & $\boldsymbol{x}_{\mathbf{4}}$ & $\boldsymbol{y}_{\mathbf{1}}$ & $\hat{\boldsymbol{y}}$ \\
\hline $\mathbf{D} \mathbf{( \mathbf { m m } )}$ & $\mathbf{H}(\mathbf{m m})$ & $\mathbf{L}(\mathbf{m m})$ & $\mathbf{R}(\mathbf{m m})$ & $\boldsymbol{u}_{\boldsymbol{L}, \boldsymbol{i}}$ & $\boldsymbol{\sigma}$ \\
\hline 40 & 75 & 15 & 90 & 0.05168 & 0.307 \\
60 & 75 & 15 & 0 & 0.78804 & 0.281 \\
20 & 75 & 15 & 50 & 0.68672 & 0.187 \\
50 & 100 & 15 & 70 & 0.15530 & 0.276 \\
30 & 50 & 0 & 0 & 0.89510 & 0.283 \\
& & & $\vdots$ & & \\
30 & 25 & 15 & 50 & 0.59876 & 0.253 \\
30 & 75 & 15 & 0 & 0.95211 & 0.239 \\
50 & 75 & 15 & 110 & 0.05073 & 0.270 \\
30 & 50 & 5 & 110 & 0.02788 & 0.301 \\
30 & 50 & 20 & 30 & 0.62597 & 0.295 \\
\hline
\end{tabular}

The operating parameters for CFD simulations and the properties of oil are shown in Table 2. The Euler gas-liquid two-phase flow model was used for the simulation, and the Schiller-Naumann drag force model was used between the two phases. The time step was $0.005 \mathrm{~s}$, and the total number of meshes was 173,800 .

Table 2. Properties of oil materials and operating parameters used.

\begin{tabular}{cc}
\hline Operating Parameters/Physical Parameter & Value \\
\hline Pressure, $\mathrm{MPa}$ & 5 \\
Hydrogen-oil ratio, $\mathrm{Nm}^{3} \cdot \mathrm{m}^{-3}$ & 706 \\
Operating temperature, ${ }^{\circ} \mathrm{C}$ & 350 \\
Liquid density, $\mathrm{kg} \cdot \mathrm{m}^{-3}$ & 694 \\
Liquid viscosity, $\mathrm{kg} \cdot \mathrm{m}^{-1} \cdot \mathrm{s}^{-1}$ & 0.00038 \\
Liquid phase velocity, $\mathrm{m} \cdot \mathrm{s}^{-1}$ & 0.23 \\
Gas phase density, $\mathrm{kg} \cdot \mathrm{m}^{-3}$ & 30.6 \\
Gas phase viscosity, $\mathrm{kg} \cdot \mathrm{m}^{-1} \cdot \mathrm{s}^{-1}$ & 0.000015 \\
Vapor velocity, $\mathrm{m} \cdot \mathrm{s}^{-1}$ & 0.24 \\
\hline
\end{tabular}

In the previous work [22] by Zhao, often only one factor was considered for each experiment or calculation, which ignores the interaction among factors. It is essential to consider the correlation between structural parameters of the gas-liquid distributor.

\subsection{Support Vector Regression and Particle Swarm Optimization}

Support vector regression (SVR) is a machine learning algorithm based on statistical theory, specifically, the principle of structural risk minimization to better solve polynomial regression problems [23,24]. In SVR, the objective function is convex, meaning that the global optimum is always reached. An abstraction of an SVR model with some kernel functions $K\left(x_{i}, x\right)$ is shown in Equation (2).

$$
f(x)=\sum_{i}^{l} \omega_{i} \times K\left(x_{i}, x\right)+b
$$

When solving a nonlinear regression problem, we actually solve for the weights $\omega_{i}$ and the threshold $b$. Our objective is to use the estimated values of $\omega_{i}$ and $b$ to minimize the regression risk by considering Equations (3) and (4).

$$
\min J=1 / 2\|\omega\|^{2}+C \sum_{i=1}^{m}\left(\xi_{i}^{*}+\xi_{i}\right)
$$




$$
\text { s.t. }\left\{\begin{array}{c}
y_{i}-(\omega \cdot \phi(x))-b \leq \varepsilon+\xi_{i} \\
(\omega \cdot \phi(x))+b-y_{i} \leq \varepsilon+\xi_{i}^{*} \\
\xi_{i}, \xi_{i}^{*} \geq 0
\end{array}\right.
$$

where $\omega$ is the weight vector, $1 / 2\|\omega\|^{2}$ is the model complexity, and $C$ is the penalty factor, $\varepsilon$ is insensitive loss, $\xi_{i}, \xi_{i}^{*}$ is the relaxation variable.

The Lagrange equation is used to solve this quadratic programming problem, and it is converted into the dual optimization corresponding to Equations (5) and (6).

$$
\begin{gathered}
\max J(\alpha)=\max \left\{-\frac{1}{2} \sum_{j=1}^{m}\left(\alpha_{i}-\alpha_{i}^{*}\right) \cdot\left(\alpha_{j}-\alpha_{j}^{*}\right) K\left(x_{i}, x_{j}\right)-\varepsilon \sum_{i=1}^{m}\left(\alpha_{i}+\alpha_{i}^{*}\right)+\sum_{i=1}^{m} y_{i}\left(\alpha_{i}-\alpha_{i}^{*}\right)\right\} \\
\text { s.t. }\left\{\begin{array}{c}
\sum_{i=1}^{m}\left(\alpha_{i}-\alpha_{i}^{*}\right)=0 \\
0 \leq \alpha_{i} \leq \mathrm{C} \\
0 \leq \alpha_{i}^{*} \leq \mathrm{C}
\end{array}\right.
\end{gathered}
$$

where $\alpha_{i}, \alpha_{i}^{*}, \alpha_{j}$ and $\alpha_{j}^{*}$ are Lagrangian operators and $K\left(x_{i}, x_{j}\right)$ is a kernel function, for which the following holds:

$$
f(x)=\sum_{i=1}^{l}\left(\alpha_{i}-\alpha_{i}^{*}\right) K\left(x_{i}, x_{j}\right)+b .
$$

The nonlinear regression function $f(x)$ is obtained through the calculation of the kernel function. The selection of the kernel function affects the non-linear mapping of the samples directly, and so choosing an appropriate kernel function is very important for SVR [25]. The Radial Basis Function (RBF) function [26] is used as the kernel function in this paper because of its excellent generalization and nonlinear regression ability. Its description is showed as below:

$$
K\left(x_{i}, x_{j}\right)=\exp \left(-\mathrm{g}\left\|x-x_{i}\right\|^{2}\right)
$$

where $g$ is a kernel function parameter.

In this study, the input and output variables of SVR model is described as Equation (9):

$$
u_{L, i}=f(D, H, L, R) .
$$

The prediction performance of SVR is closely related to the RBF kernel parameter $g$, the penalty factor $C$, and the insensitive loss $\varepsilon$ [27]. In order to obtain a support vector regression machine with better performance, particle swarm optimization (PSO) is adopted for parameter optimization in this paper. Five-fold cross-validation [28] is used to evaluate the fitness of each particle in the PSO algorithm to improve the model prediction performance. It is essential to set a lower limit for the root mean square error (RMSE) in the PSO optimization process to prevent the SVR model from overfitting. When the RMSE is less than the lower limit, the optimization process is ended. The calculation formula of RMSE is as follows:

$$
\text { RMSE }=\sqrt{\frac{1}{n} \sum_{i=1}^{n}\left(y_{i}-f\left(x_{i}\right)\right)^{2}}
$$

where $n$ is the number of samples; $y_{i}$ is the true value and $f\left(x_{i}\right)$ is the predicted value of the model. The PSO algorithm aims to minimize the overall error of the model.

After 60 iterations, the RMSE of the training set after 5-fold cross-validation is $1.215 \times 10^{-3}$, and the optimization results of $c, g$, and $\varepsilon$ are $163,0.737$, and 0.241 , respectively. The training set and the optimized $C, g$, and $\varepsilon$ values were then applied to the SVR model for training. Then, the test set was input to the trained SVR model to obtain the corresponding predicted value. Finally, the performance of the model was evaluated by comparing the prediction values and the real values. 


\subsection{Evaluation Index}

The validity of the PSO-SVR model was evaluated by comparing the accuracy and rapidity of the algorithm. For comparison purposes, the normalized mean square error (MSE) and the coefficient of determination $\left(R^{2}\right)$ were used as performance indicators.

$$
\begin{aligned}
M S E & =\frac{1}{n} \sum_{i=1}^{n}\left(y_{i}-f\left(x_{i}\right)\right)^{2} \\
\mathbf{R}^{2} & =\frac{\sum_{i=1}^{n}\left(f\left(x_{i}\right)-\bar{y}\right)^{2}}{\sum_{i=1}^{n}\left(y_{i}-\bar{y}\right)^{2}}
\end{aligned}
$$

where $n$ is the number of samples; $y_{i}$ is the true value; $f\left(x_{i}\right)$ is the value predicted by the model; and $\bar{y}$ is the average of the true values. A smaller MSE is indicative of higher model prediction accuracy. $R^{2}$ ranges from 0 to 1 and is the degree of correlation between the experimental and predictive values. A higher $R^{2}$ value corresponds to better agreement between observed and simulated results. Here, a higher $R^{2}$ value and a lower MSE value indicate a better prediction performance.

\section{Optimal Structure Obtained from RSM Based on the PSO-SVR Model}

The data obtained using the PSO-SVR hybrid model were applied as the input for the response surface methodology (RSM) to obtain the optimal structural parameters of the distributor.

Response Surface Methodology

RSM is a simple model-based mathematical tool with a low computational burden for predicting and optimizing engineering problems [29]. It mainly uses a reasonable experimental design method and obtains a polynomial equation to describe the behavior of a dataset with the objective of making statistical previsions, while a neural network and PSO cannot be used for such a formulation.

In this article, three structural parameters, namely throat diameter $\mathrm{D}$, throat height $\mathrm{H}$, and throat length L in a Venturi gas-liquid distributor, were selected as influencing factors. The domain of the three structural parameters was determined according to the structure of hydrogenation reactor [22]. The maximum and minimum values of the three factors were input to the Design-Expert design software. 53 structural combinations of the three factors and five levels were obtained by user-defined experimental design. The PSO-SVR model was used as the proxy model to obtain the liquid maldistribution factor $\sigma$ corresponding to various structural combinations of the Venturi gas-liquid distributor, as shown in Table 3.

Table 3. Experimental plan designed by response surface methodology (RSM) and corresponding responses.

\begin{tabular}{ccccc}
\hline Sample No. & Throat Diameter, D & Throat Height, H & Throat Length, L & $\begin{array}{c}\text { Response (Liquid } \\
\text { Maldistribution Factor, } \boldsymbol{\sigma} \text { ) }\end{array}$ \\
\hline 1 & 40 & 100 & 20 & 0.3767 \\
2 & 20 & 100 & 10 & 0.2729 \\
3 & 40 & 62.5 & 15 & 0.2502 \\
4 & 60 & 25 & 20 & 0.3629 \\
5 & 50 & 43.75 & 15 & 0.2499 \\
6 & 30 & 81.25 & 5 & 0.3113 \\
7 & 62.5 & 0 & 0.2621 \\
& 20 & & & \\
49 & & 25 & 0 & 0.3082 \\
50 & 40 & 62.5 & 10 & 0.3656 \\
51 & 60 & 43.75 & 10 & 0.3376 \\
53 & 50 & 62.5 & 15 & 0.2355 \\
53 & 100 & 20 & 0.2663 \\
\hline
\end{tabular}


The quartic regression, Equation (13), was applied to find the interaction relationship between the three structural parameters and the liquid maldistribution factor as follows:

$$
\begin{array}{cc}
\hat{\sigma}=+0.34+\quad 0.088 \times \mathrm{D}+0.069 \times \mathrm{H}-0.14 \times \mathrm{L}+0.1 \times \mathrm{D} \times \mathrm{H}-0.11 \times \mathrm{D} \times \mathrm{L} \\
& -0.056 \times \mathrm{H} \times \mathrm{L}-0.13 \times \mathrm{L}^{2}-0.059 \times \mathrm{D}^{2} \times \mathrm{H}-0.015 \times \mathrm{D}^{2} \times \mathrm{L} \\
& -0.037 \times \mathrm{D} \times \mathrm{H}^{2}-0.031 \times \mathrm{D}^{3}-0.038 \times \mathrm{H}^{3}+0.15 \times \mathrm{L}^{3} \\
& +0.027 \times \mathrm{D}^{2} \times \mathrm{L}^{2}-0.072 \times \mathrm{D}^{3} \times \mathrm{H}+0.1 \times \mathrm{D}^{3} \times \mathrm{L} \\
& +0.049 \times \mathrm{H}^{3} \times \mathrm{L}-0.018 \times \mathrm{D}^{4}+0.026 \times \mathrm{H}^{4}+0.12 \times \mathrm{L}^{4}
\end{array}
$$

Normally, the analysis of variance (ANOVA) is considered a very important tool in finding the best fitting mathematical regression model. Table 4 shows a valid model obtained from ANOVA. The $p$-value of the model obtained in this experiment was far less than 0.05 , which indicates that the obtained regression equation is statistically significant.

Table 4. Analysis of variance (ANOVA) for liquid maldistribution by quartic modeling.

\begin{tabular}{cccccc}
\hline Source & Sum of Squares & $\mathbf{d f}$ & Mean Square & F Value & $\begin{array}{c}p \text {-Value } \\
\text { Prob }>\text { F }\end{array}$ \\
\hline Model & 0.17 & 20 & $8.482 \times 10^{-3}$ & 43.41 & $<0.0001$ \\
$\mathrm{D}$ & 0.019 & 1 & 0.019 & 98.37 & $<0.0001$ \\
$\mathrm{H}$ & 0.012 & 1 & 0.012 & 60.36 & $<0.0001$ \\
$\mathrm{~L}$ & 0.049 & 1 & 0.049 & 250.70 & $<0.0001$ \\
$\mathrm{D} \times \mathrm{H}$ & $4.524 \times 10^{-3}$ & 1 & $4.524 \times 10^{-3}$ & 23.15 & $<0.0001$ \\
$\mathrm{D} \times \mathrm{L}$ & $5.073 \times 10^{-3}$ & 1 & $5.073 \times 10^{-3}$ & 25.96 & $<0.0001$ \\
$\mathrm{H} \times \mathrm{L}$ & $1.341 \times 10^{-3}$ & 1 & $1.341 \times 10^{-3}$ & 6.86 & 0.0133 \\
$\mathrm{~L}^{2}$ & $4.157 \times 10^{-3}$ & 1 & $4.157 \times 10^{-3}$ & 21.28 & $<0.0001$ \\
$\mathrm{D}^{2} \times \mathrm{H}$ & 0.014 & 1 & 0.014 & 73.31 & $<0.0001$ \\
$\mathrm{D}^{2} \times \mathrm{L}$ & $9.267 \times 10^{-4}$ & 1 & $9.267 \times 10^{-4}$ & 4.74 & 0.0369 \\
$\mathrm{D} \times \mathrm{H}^{2}$ & $5.669 \times 10^{-3}$ & 1 & $5.669 \times 10^{-3}$ & 29.01 & $<0.0001$ \\
$\mathrm{D}^{3}$ & $1.549 \times 10^{-3}$ & 1 & $1.549 \times 10^{-3}$ & 7.93 & 0.0083 \\
$\mathrm{H}^{3}$ & $2.348 \times 10^{-3}$ & 1 & $2.348 \times 10^{-3}$ & 12.01 & 0.0015 \\
$\mathrm{~L}^{3}$ & 0.035 & 1 & 0.035 & 180.89 & $<0.0001$ \\
$\mathrm{D}^{2} \times \mathrm{L}^{2}$ & $1.281 \times 10^{-3}$ & 1 & $1.281 \times 10^{-3}$ & 6.55 & 0.0154 \\
$\mathrm{D}^{3} \times \mathrm{H}$ & $2.071 \times 10^{-3}$ & 1 & $2.071 \times 10^{-3}$ & 10.60 & 0.0027 \\
$\mathrm{D}^{3} \times \mathrm{L}$ & $4.035 \times 10^{-3}$ & 1 & $4.035 \times 10^{-3}$ & 20.65 & $<0.0001$ \\
$\mathrm{H}^{3} \times \mathrm{L}$ & $9.451 \times 10^{-4}$ & 1 & $9.451 \times 10^{-4}$ & 4.84 & 0.0352 \\
$\mathrm{D}^{4}$ & $1.013 \times 10^{-3}$ & 1 & $1.013 \times 10^{-3}$ & 5.19 & 0.0296 \\
\hline
\end{tabular}

Table 5 shows the statistical analysis of the regression equation's error. $R^{2}=0.9644$ indicates that there is a statistical goodness of fit between the model and the experimental data. The good agreement between the predicted $R^{2}$ value of 0.8981 and the adjusted $R^{2}$ value of 0.9422 indicates that the model gives a good estimation of response in the studied range. The coefficient of variation (CV) was $4.22 \%$, i.e., less than $10 \%$, which is indicative of the high reliability and accuracy of the regression equation. As a ratio of signal effectiveness, our Adeq Precision of 27.651 was greater than 4, which demonstrates the high rationality of the regression equation.

Table 5. Statistical analysis of regression equation errors.

\begin{tabular}{cc}
\hline Statistics Project & Value \\
\hline Std. Dev. & 0.014 \\
Mean & 0.33 \\
CV \% & 4.22 \\
PRESS & 0.018 \\
R-Squared & 0.9644 \\
Adj R-Squared & 0.9422 \\
Pred R-Squared & 0.8981 \\
Adeq Precision & 27.651 \\
\hline
\end{tabular}


The 3D response surface plots for the liquid maldistribution factor illustrate the influence of structural parameters and the interactions on the response, as shown in Figure 3. The combined effects of $\mathrm{D}$ and $\mathrm{H}, \mathrm{D}$ and $\mathrm{L}, \mathrm{H}$ and $\mathrm{L}$ on liquid maldistribution factor $\sigma$ were depicted in Figure $3 \mathrm{a}-\mathrm{c}$, respectively. Liquid maldistribution factor $\sigma$ decreases with the increase of throat length $L$ and the decrease of throat diameter $\mathrm{D}$. The response surface results show that the longer throat length $\mathrm{L}$ and the smaller throat diameter $\mathrm{D}$, the better it is to reduce liquid maldistribution $\sigma$, while the impact of throat height $\mathrm{H}$ is less significant.

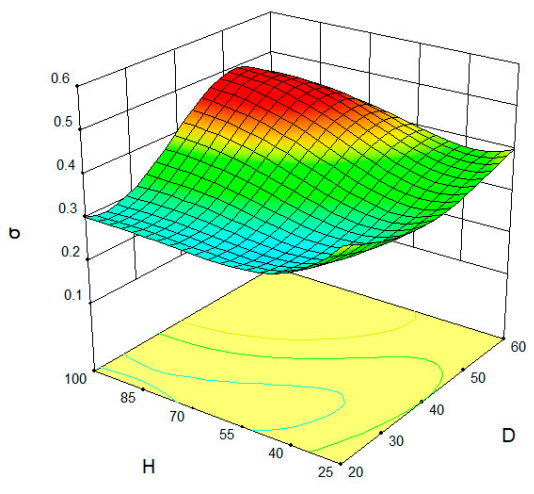

(a)

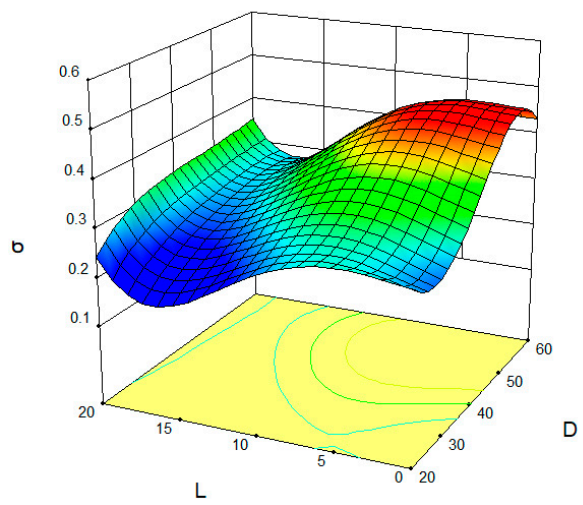

(b)

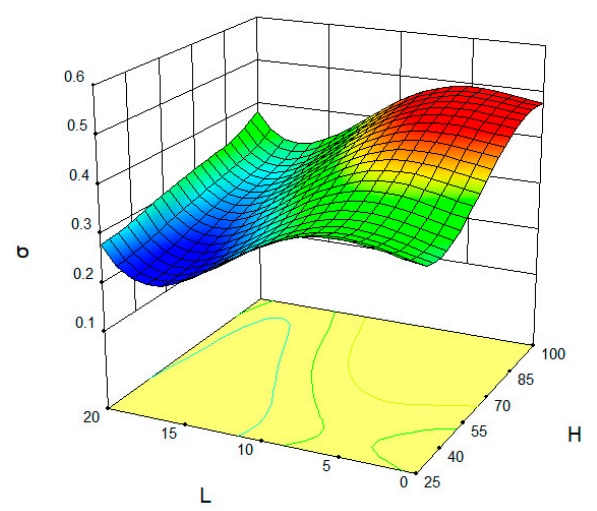

(c)

Figure 3. 3D plot of response surface for liquid maldistribution factor $\sigma$. (a) Throat diameter D and throat height $\mathrm{H}$ combination; (b) throat diameter D and throat length L combination; (c) throat height $\mathrm{H}$ and throat length $\mathrm{L}$ combination.

The RSM optimization was carried out to minimize the liquid maldistribution factor. The optimal response combination was obtained for a throat diameter D of $20 \mathrm{~mm}$, a throat height $\mathrm{H}$ of $84.5 \mathrm{~mm}$, and a throat length $\mathrm{L}$ of $16.4 \mathrm{~mm}$, and a liquid maldistribution factor $\sigma$ of 0.162 . For comparison, the optimal structure proposed by Zhao was $\mathrm{D}=30 \mathrm{~mm}, \mathrm{H}=50 \mathrm{~mm}, \mathrm{~L}=15 \mathrm{~mm}$, and $\sigma=0.184$. The optimal estimate of the liquid maldistribution factor in this paper is lower than reported by Zhao et al.

\section{Results and Discussion}

\subsection{Effectiveness Evaluation of the PSO-SVR Model}

The validity of the PSO-SVR model as the proxy model was evaluated by comparing the accuracy rapidity of the algorithm. 


\subsubsection{Comparison and Discussion on the Precision of PSO-SVR Model}

Figure 4 shows the prediction results of training and test samples of the PSO-SVR model. The abscissa represents the simulation value of the apparent linear velocity of the liquid obtained using CFD [22], and the ordinate represents the output predicted value of the PSO-SVR model. The samples of the training set and the test set are all concentrated in the diagonal attachment, which indicates that the prediction results are consistent with the experimental data. As shown in Figure 4, the MSE of the PSO-SVR model for the training set was $2.364 \times 10^{-4}$ and the coefficient of determination $\mathrm{R}^{2}$ was 0.991 , which shows a high training accuracy. The normalized MSE of the test set was $5.391 \times 10^{-4}$ and the coefficient of determination $\mathrm{R}^{2}$ was 0.985 , which shows that the prediction result is still good. Therefore, the PSO-SVR model can be used as a proxy model to predict the liquid distribution of the gas-liquid distributor accurately.

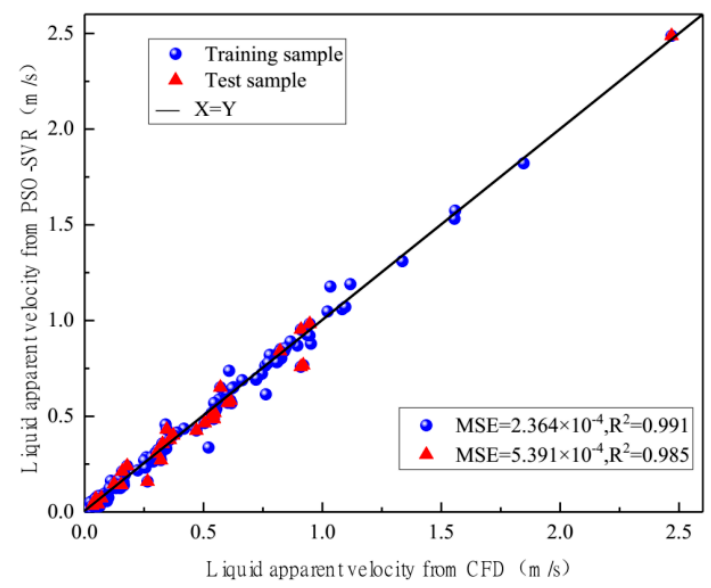

Figure 4. The prediction results of training and test samples.

\subsubsection{Comparison and Discussion on the Rapidity of PSO-SVR Model}

The comparison result of the SVR algorithm optimized with the grid search method and the PSO-SVR model is shown in Table 6. Optimizing the SVR parameters using PSO yielded a result of $\{C=163, g=0.737, \varepsilon=0.241\}$, while optimization using the grid search method resulted in $\{C=5.6569$, $g=1.071, \varepsilon=0.01\}$. The MSE of the PSO-SVR model was $5.391 \times 10^{-4}$, and $\mathrm{R}^{2}$ was 0.985 , while the MSE of the SVR model was $1.314 \times 10^{-3}$, and $\mathrm{R}^{2}$ was 0.976 . The CPU time required by the SVR with the standard grid method and PSO-SVR $(t=1047 \mathrm{~s}$ and $36.15 \mathrm{~s}$, respectively). The results show that, as a very effective evolutionary algorithm, the PSO can replace the standard grid search method to find better model parameters and improve the optimization speed and accuracy.

Table 6. Comparison of evaluation parameters between particle swarm optimization-support vector regression (PSO-SVR) and support vector regression (SVR).

\begin{tabular}{ccccc}
\hline & $\{C, g, \varepsilon\}$ & MSE & $\mathbf{R}^{2}$ & CPU Time \\
\hline PSO-SVR & $\{C=163, g=0.737, \varepsilon=0.241\}$ & $5.391 \times 10^{-4}$ & 0.985 & $36.15 \mathrm{~s}$ \\
SVR & $\{C=5.6569, g=1.071, \varepsilon=0.01\}$ & $1.314 \times 10^{-3}$ & 0.976 & $1047 \mathrm{~s}$ \\
\hline
\end{tabular}

\subsection{Verification of the Optimal Structure}

To verify the credibility of the optimal structure of the gas-liquid distributor proposed in this paper, CFD simulations were conducted using ANSYS FLUENT V15.00 and run on a high-performance computing (HPC) system. The computations were carried out using an Intel Xeon $(R)$ Gold 5118 processor with $24 \mathrm{CPU}$ cores. As far as the boundary conditions are concerned, velocity-inlet conditions were used at the domain inlet and a pressure outlet condition was specified at the domain exit. 
The physical properties and initial speed were set according to the properties of oil materials and operating parameters in Table 2. The input speed of the single distributor was $0.02987 \mathrm{~m} / \mathrm{s}$, and the cylindrical wall surface was set as an adiabatic, non-slip wall surface. The standard $k-\varepsilon$ model was selected as the turbulence model, using the SIMPLE-algorithm for the pressure-velocity coupling. The 3D non-steady state was taken as the initial condition for calculation. With a time step of $0.005 \mathrm{~s}$, converge was achieved at $150 \mathrm{~s}$ for residual curve values of all the equations constantly stable below 0.001 .

The velocity distribution contour of the gas-liquid distributor converging to steady state is shown in Figure 5. The velocity distribution of the oil materials at $300 \mathrm{~mm}$ below the gas-liquid distributor tray is obtained as $1.4633,1.3517,1.0972,0.7715,0.4911,0.2405$ at the sampling position mentioned in Section 2.1. Maldistribution factor $\sigma$ was calculated as 0.159 according to Equation (1), which is in good agreement with the value of 0.162 obtained using RSM.
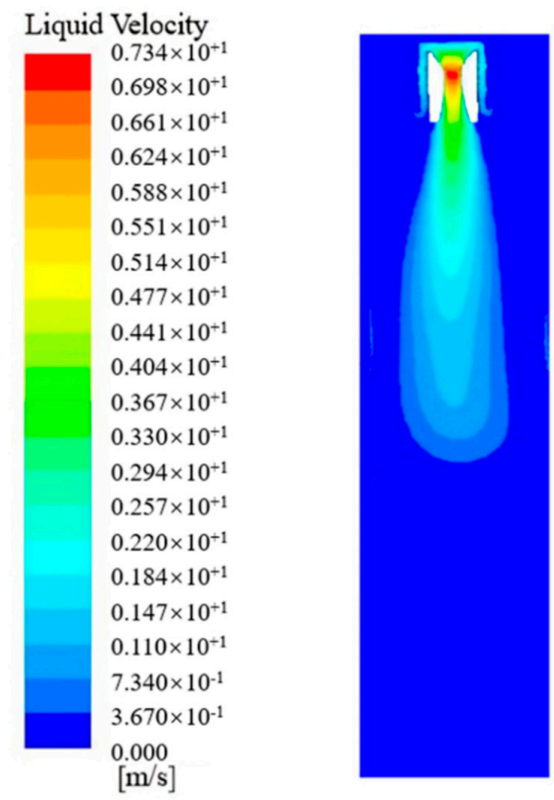

Figure 5. Velocity contour of Venturi distributor at central surface.

The distribution performance of the distributor with the optimal structure is analyzed according to the liquid velocity distribution contour shown in Figure 5. After falling into the distribution tray, the materials continue to pile up, and then enter the dispenser under the suction of the slit. In this process, the gas and liquid phases are violently mixed. While the continuous liquid phase is broken by the gas phase, it flows upward along the channel, and the materials in different directions crash above the downcomer and flow downward. Due to the improved Venturi structure existing in the gas-liquid distributor, the flow area at the throat position decreases sharply, with the velocity of oil materials reaching a maximum speed of $7.34 \mathrm{~m} / \mathrm{s}$. The accumulation of materials in the position of the throat causes a large pressure above the throat, and a high-pressure difference is forming under the throat. The material fills the outlet section quickly when it flows out through the throat under the effect of the pressure difference. The inclined outlet section in the Venturi structure produces a larger diffusion angle when the material flows out of the distributor, thus a larger radial distribution of the material on the catalyst bed is achieved.

\section{Conclusions}

In this study, we investigate the utility of using the PSO algorithm for optimizing the parameters of SVR in order to obtain an effective hybrid model (PSO-SVR) for application in modeling liquid maldistribution of the gas-liquid distributor in TBRs. The hybrid PSO-SVR model was based on limited CFD simulation data to replace the time-consuming CFD simulation for different cases, with a very 
low time cost for accurately predicting the results of the liquid distribution. RSM was used to predict the optimal structure of the gas-liquid distributor in TBRs based on the PSO-SVR model. To ensure the accurate values of the best structural parameters obtained using RSM, another simulation of these structural was performed using CFD to determine the liquid maldistribution. The CFD simulation results reaffirmed the accuracy of the optimal structure proposed in this paper.

The model development, experiment design, and CFD validation are coupled with the following concluding remarks:

(1) SVR was used with limited CFD simulation data to overcome the high computational load issue of CFD and achieve an accurate mathematical model.

(2) The PSO algorithm was employed to determine an optimal set of parameters of the SVR model and speed up modeling.

(3) The optimal structure of gas-liquid distributor was obtained from the combination of PSO-SVR and RSM.

(4) RSM was applied to clarify the influence of structural parameters of the gas-liquid distributor on the liquid maldistribution.

(5) A closed-loop optimized system was designed beginning from collecting CFD data, modeling with PSO-SVR, optimizing by RSM, and ending with CFD validation.

The closed-loop optimized system proposed in this manuscript could also be applied to the modeling and optimization of chemical process, as well as related chemical equipment structure.

Author Contributions: Conceptualization, W.Z. and L.L.; methodology, W.Z.; software, L.L.; validation, W.Z., L.L., and B.Z.; investigation, X.X.; data curation, J.Z.; writing-original draft preparation, W.Z. and L.L.; writing-review and editing, W.Z.; project administration, W.Z.; funding acquisition, W.Z. and J.W. All authors have read and agreed to the published version of the manuscript.

Funding: This research was funded by National Key Research and Development Program of China (No. 2018YFB0604603-03), Key Research and Development Plan Projects of Shanxi Province (No. 201903D121027), National Natural Science Foundation of China (No. 21978189).

Conflicts of Interest: The authors declare no conflict of interest.

\section{References}

1. Atta, A.; Roy, S.; Nigam, K.D.P. Investigation of liquid maldistribution in trickle-bed reactors using porous media concept in CFD. Chem. Eng. Sci. 2007, 62, 7033-7044. [CrossRef]

2. Marcandelli, C.; Lamine, A.S.; Bernard, J.R.; Wild, G. Liquid Distribution in Trickle-Bed Reactor. Oil Gas. Sci. Technol. 2000, 55, 407-415. [CrossRef]

3. Kundu, A.; Saroha, A.K.; Nigam, K.D.P. Liquid distribution studies in trickle-bed reactors. Chem. Eng. Sci. 2001, 56, 5963-5967. [CrossRef]

4. Li, M.; Iida, N.; Yasuda, K.; Bando, Y.; Nakamura, M. Effect of orientation of packing structure on liquid flow distribution in trickle bed. J. Chem. Eng. Jpn. 2000, 33, 811-814. [CrossRef]

5. Zalucky, J.; Wagner, M.; Schubert, M.; Lange, R.; Hampel, U. Hydrodynamics of descending gas-liquid flows in solid foams: Liquid holdup, multiphase pressure drop and radial dispersion. Chem. Eng. Sci. 2017, 168, 480-494. [CrossRef]

6. Roy, S.; Kamalanathan, P.; Al-Dahhan, M. Integration of phase distribution from gamma-ray tomography technique with monolith reactor scale modeling. Chem. Eng. Sci. 2019, 200, 27-37. [CrossRef]

7. Wu, H.; Buschle, B.; Yang, Y.; Tan, C.; Dong, F.; Jia, J.; Lucquiaud, M. Liquid distribution and hold-up measurement in counter current flow packed column by electrical capacitance tomography. Chem. Eng. J. 2018, 353, 519-532. [CrossRef]

8. Singh, B.K.; Jain, E.; Buwa, V.V. Feasibility of Electrical Resistance Tomography for measurements of liquid holdup distribution in a trickle bed reactor. Chem. Eng. J. 2019, 358, 564-579. [CrossRef]

9. Lovreglio, P.; Das, S.; Buist, K.A.; Peters, E.A.J.F.; Pel, L.; Kuipers, J.A.M. Experimental and numerical investigation of structure and hydrodynamics in packed beds of spherical particles. AIChE J. 2018, 64, 1896-1907. [CrossRef] 
10. Uribe, S.; Cordero, M.E.; Reyes, E.P.; Regalado-Méndez, A.; Zárate, L.G. Multiscale CFD modelling and analysis of TBR behavior for an HDS process: Deviations from ideal behaviors. Fuel 2019, 239, 1162-1172. [CrossRef]

11. He, G.; Mo, H.; Zhang, R.; Jin, H.; Du, W. Study of the effects of walls on vortex formation and liquid maldistribution with two-phase flow around a spherical particle via numerical simulation. Powder Technol. 2019, 354, 125-135. [CrossRef]

12. Shah, M.T.; Utikar, R.P.; Pareek, V.K. Effect of column inclination and oscillation on liquid spreading in a trickle bed. Chem. Eng. Res. Des. 2019, 152, 165-179. [CrossRef]

13. Klenov, O.P.; Noskov, A.S. Influence of input conditions on the flow distribution in trickle bed reactors. Chem. Eng. J. 2020, 382. [CrossRef]

14. Dhanraj, D.I.A.; Buwa, V.V. Effect of capillary pressure force on local liquid distribution in a trickle bed. Chem. Eng. Sci. 2018, 191, 115-133. [CrossRef]

15. Mehmani, A.; Chowdhury, S.; Meinrenken, C.; Messac, A. Concurrent surrogate model selection (COSMOS): Optimizing model type, kernel function, and hyper-parameters. Struct. Multidiscip. Optim. 2018, 57, 1093-1114. [CrossRef]

16. Cheng, K.; Lu, Z. Adaptive sparse polynomial chaos expansions for global sensitivity analysis based on support vector regression. Comput. Struct. 2018, 194, 86-96. [CrossRef]

17. Xiang, H.; Li, Y.; Liao, H.; Li, C. An adaptive surrogate model based on support vector regression and its application to the optimization of railway wind barriers. Struct. Multidiscip. Optim. 2017, 55, 701-713. [CrossRef]

18. Zhang, W.; Zhang, L.; Yang, J.; Hao, X.; Guan, G.; Gao, Z. An experimental modeling of cyclone separator efficiency with PCA-PSO-SVR algorithm. Powder Technol. 2019, 347, 114-124. [CrossRef]

19. Bansal, S.; Roy, S.; Larachi, F. Support vector regression models for trickle bed reactors. Chem. Eng. J. 2012, 207-208, 822-831. [CrossRef]

20. Jalalifar, S.; Masoudi, M.; Abbassi, R.; Garaniya, V.; Ghiji, M.; Salehi, F. A hybrid SVR-PSO model to predict a CFD-based optimised bubbling fluidised bed pyrolysis reactor. Energy 2020, 191. [CrossRef]

21. Zaidi, S. Development of support vector regression (SVR)-based model for prediction of circulation rate in a vertical tube thermosiphon reboiler. Chem. Eng. Sci. 2012, 69, 514-521. [CrossRef]

22. Zhao, F. Study on Fluid Flow Characteristics and Lumped Reaction Kinetics in Hydrogenation Reactor; China University of Petroleum: Beijing, China, 2016.

23. Tabari, M.M.R.; Sanayei, H.R.Z. Prediction of the intermediate block displacement of the dam crest using artificial neural network and support vector regression models. Soft Comput. 2019, 23, 9629-9645. [CrossRef]

24. Yang, H.; Nikafshan Rad, H.; Hasanipanah, M.; Bakhshandeh Amnieh, H.; Nekouie, A. Prediction of Vibration Velocity Generated in Mine Blasting Using Support Vector Regression Improved by Optimization Algorithms. Nat. Resour. Res. 2020, 29, 807-830. [CrossRef]

25. Hasanipanah, M.; Shahnazar, A.; Bakhshandeh Amnieh, H.; Jahed Armaghani, D. Prediction of air-overpressure caused by mine blasting using a new hybrid PSO-SVR model. Eng. Comput. 2017, 33, 23-31. [CrossRef]

26. Akinpelu, A.A.; Ali, M.E.; Owolabi, T.O.; Johan, M.R.; Saidur, R.; Olatunji, S.O.; Chowdbury, Z. A support vector regression model for the prediction of total polyaromatic hydrocarbons in soil: An artificial intelligent system for mapping environmental pollution. Neural Comput. Appl. 2020, 3. [CrossRef]

27. Zuo, Q.; Zhu, X.; Liu, Z.; Zhang, J.; Wu, G.; Li, Y. Prediction of the performance and emissions of a spark ignition engine fueled with butanol-gasoline blends based on support vector regression. Environ. Prog. Sustain. Energy 2019, 38, 1-9. [CrossRef]

28. Chen, H.; Liu, C.; Xu, X.; Zhang, L. A new model for predicting sulfur solubility in sour gases based on hybrid intelligent algorithm. Fuel 2020, 262, 116550. [CrossRef]

29. Keshtegar, B.; Heddam, S.; Sebbar, A.; Zhu, S.P.; Trung, N.T. SVR-RSM: A hybrid heuristic method for modeling monthly pan evaporation. Environ. Sci. Pollut. Res. 2019, 26, 35807-35826. [CrossRef]

Publisher's Note: MDPI stays neutral with regard to jurisdictional claims in published maps and institutional affiliations. 